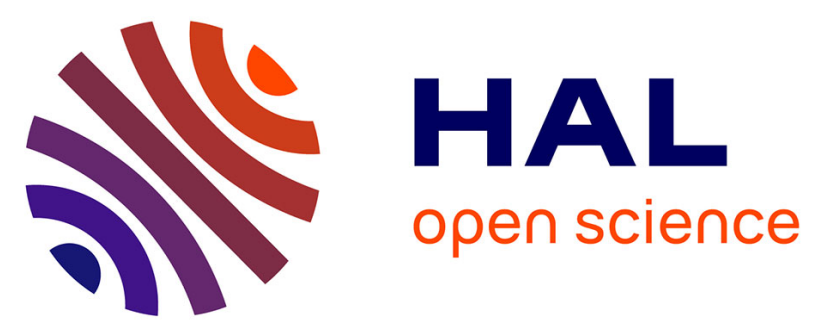

\title{
Anisotropic super-attenuation of capillary waves on driven glass interfaces
}

Bruno Bresson, Coralie Brun, Xavier Buet, Yong Chen, Matteo Ciccotti, Jérôme Gâteau, Greg Jasion, Marco Petrovich, Francesco Poletti, David Richardson, et al.

\section{To cite this version:}

Bruno Bresson, Coralie Brun, Xavier Buet, Yong Chen, Matteo Ciccotti, et al.. Anisotropic superattenuation of capillary waves on driven glass interfaces. Physical Review Letters, 2017, 119 (23), pp.235501 10.1103/PhysRevLett.119.235501 . hal-01532487v2

\section{HAL Id: hal-01532487 \\ https://hal.science/hal-01532487v2}

Submitted on 6 Dec 2017

HAL is a multi-disciplinary open access archive for the deposit and dissemination of scientific research documents, whether they are published or not. The documents may come from teaching and research institutions in France or abroad, or from public or private research centers.
L'archive ouverte pluridisciplinaire HAL, est destinée au dépôt et à la diffusion de documents scientifiques de niveau recherche, publiés ou non, émanant des établissements d'enseignement et de recherche français ou étrangers, des laboratoires publics ou privés. 


\title{
Anisotropic Superattenuation of Capillary Waves on Driven Glass Interfaces
}

\author{
Bruno Bresson, ${ }^{1}$ Coralie Brun, ${ }^{2}$ Xavier Buet, ${ }^{2}$ Yong Chen, ${ }^{3}$ Matteo Ciccotti, ${ }^{1}$ Jérôme Gâteau, ${ }^{4}$ Greg Jasion, ${ }^{3}$ \\ Marco N. Petrovich, ${ }^{3}$ Francesco Poletti, ${ }^{3}$ David J. Richardson, ${ }^{3}$ Seyed Reza Sandoghchi, ${ }^{3}$ Gilles Tessier, ${ }^{4}$ \\ Botond Tyukodi, ${ }^{2,5}$ and Damien Vandembroucq ${ }^{2}$ \\ ${ }^{1}$ SIMM, ESPCI Paris/CNRS-UMR 7615/Université Paris 6 UPMC/PSL Research University, \\ 10 rue Vauquelin, 75231 Paris cedex 05, France \\ ${ }^{2}$ PMMH, ESPCI Paris/CNRS-UMR 7636/Université Paris 6 UPMC/Université Paris 7 Diderot/PSL Research University, \\ 10 rue Vauquelin, 75231 Paris cedex 05, France \\ ${ }^{3}$ Optoelectronics Research Center, University of Southampton, Highfields, Southampton SO17 1BJ, United Kingdom \\ ${ }^{4}$ Neurophotonics Lab, CNRS UMR 8250, Université Paris Descartes, 45 rue des Saints Pères, Paris, France \\ ${ }^{5}$ Physics department, University Babeş-Bolyai, 1 str. Mihail Kogălniceanu, 400084 Cluj-Napoca, Romania
}

(Received 31 May 2017; published 5 December 2017)

\begin{abstract}
Metrological atomic force microscopy measurements are performed on the silica glass interfaces of photonic band-gap fibers and hollow capillaries. The freezing of attenuated out-of-equilibrium capillary waves during the drawing process is shown to result in a reduced surface roughness. The roughness attenuation with respect to the expected thermodynamical limit is determined to vary with the drawing stress following a power law. A striking anisotropic character of the height correlation is observed: glass surfaces thus retain a structural record of the direction of the flow to which the liquid was submitted.
\end{abstract}

DOI: 10.1103/PhysRevLett.119.235501

What governs the structure of a glass surface? To very good approximation, the bulk structure of a vitreous material resembles a snapshot of the liquid before glass transition [1]. Similarly, the surface of a glass corresponds to the frozen liquid interface [2,3], and can reveal frozen surface modes of this interface. Over a wide range of length scales, from the nanometer up to the millimeter range, the subnanometer roughness of a fire-polished glass surface results from the superposition of frozen thermal equilibrium capillary waves of the liquid [4,5].

At equilibrium, capillary fluctuations of liquid interfaces originate from the interplay between thermal noise $\left(k_{B} T\right)$ and interface tension $(\gamma)$, and result in a superposition of capillary waves. Height fluctuations scale as $w_{0}=\sqrt{k_{B} T / \gamma}$ which equals $0.3-0.4 \mathrm{~nm}$ for most simple liquids. Liquid interfaces are thus extremely smooth.

Thermal interface fluctuations correspond to a lower bound of the interface width imposed by equilibrium thermodynamics. In this context, the application of any external field is usually expected to enhance the level of fluctuations, e.g., hydrodynamic instabilities [6]. However, recent results suggest that shear flow may in fact induce a nonlinear attenuation of capillary waves [7-9].

Here we present an accurate experimental characterization of such an attenuation of capillary fluctuations on glass surfaces. In particular, we show that a glass surface retains a structural record of the direction of the flow to which the liquid was submitted. Performing high precision atomic force microscopy (AFM) roughness measurements on the inner interfaces of photonic band-gap fibers and hollow capillaries produced by fiber drawing, we show that driven glass interfaces result from the freezing of attenuated capillary waves. Out-of-equilibrium interface fluctuations are strongly anisotropic with an overall amplitude that presents a nonlinear attenuation with respect to the expected equilibrium thermodynamic limit.

Measurements of capillary waves.-Capillary fluctuations at interfaces of equilibrium and supercooled liquids have been extensively studied by light scattering techniques $[3,10-15]$ but have long been difficult to measure in the real space. Direct optical observation could be achieved through the use of a near critical colloid-polymer mixture characterized by an ultralow interface tension [16], but cannot access easily to the full spatiotemporal spectrum of thermal capillary waves.

It is, however, possible to restrict the scope of the study and to access quantitatively either the temporal or the spatial part of the interface fluctuations. Interferometer-based surface fluctuation specular reflection (SFSR) has recently allowed the frequency spectrum of thermal capillary waves to be probed on thin films $[17,18]$. Spatial fluctuations of liquid interfaces can be accessed in a very different context: glass surfaces. When cooled down from the liquid state through a glass transition, equilibrium thermal capillary waves suddenly get frozen in. The intrinsic roughness of glass surfaces thus directly results from the spatial fluctuations of capillary waves frozen at the glass transition temperature $T_{G}$ [2]. Thorough AFM measurements on glass surfaces can be used to quantitatively extract the surface structure factor $S_{0}(\vec{q})$ associated with such frozen capillary waves $[4,5]$ :

$$
S_{0}(\vec{q})=\frac{k_{B} T_{G}}{\gamma|\vec{q}|^{2}}
$$


where $\vec{q}$ is the wave vector of the capillary waves [19], $k_{B}$ the Boltzmann constant, and $\gamma$ the interface tension.

The quantitative characterization of the roughness spectrum of glass interfaces has recently been the subject of a growing interest in the context of the development of hollow-core photonic band-gap fibers (HC-PBGF) [4,20-22]. In such microstructured fibers, light propagates through air within the hollow core and the ultimate losses are expected to be determined by the scattering from the inner interfaces of the fiber [23].

In the following, we first report AFM measurements on inner silica glass interfaces of HC-PBGFs [24,35]. They show significantly lower roughness levels than the expected lower bound, i.e., frozen capillary waves obtained on the surface of the same amorphous material before drawing. Moreover, the surface roughness appears to be highly anisotropic. By analogy with liquid interfaces under shear flow, we then perform AFM measurements on the inner surfaces of hollow fibers obtained in different drawing conditions and we provide evidence for a nonlinear attenuation effect controlled by the viscous stress experienced by the fibers during drawing.

Attenuated frozen capillary waves on inner glass interfaces of photonic band-gap fibers.-Surface roughness was measured on the core interface of a 37 cell HC-PBGF. Optical fibers were obtained using a two-step stack and draw process [22,36]. In a first step, cylindrical fused silica capillaries of millimetric diameter were stacked in a triangular lattice with 37 capillaries omitted to form the inner hollow core. The assembly was then drawn into $\sim 1 \mathrm{~m}$ lengths cane of a few $\mathrm{mm}$ diameter. In a second step, the cane was inserted into a sleeve jacket and the assembly drawn down to optical fiber dimensions with a diameter of the order of $100 \mu \mathrm{m}$.

As illustrated in Fig. 1, during the drawing process of the centimetric diameter preform into a fiber, the silica glass is heated up to about $2000{ }^{\circ} \mathrm{C}$ in a furnace where it reaches the liquid state and flows under the action of a pulling force, before experiencing a sudden quench to below the glass transition temperature as it exits the furnace. Out-ofequilibrium capillary fluctuations are thus frozen on the air-glass interfaces of the fiber microstructure. Insets in Fig. 1 illustrate the surface roughness on the inner surface of the final drawn hollow core and of the fire polished silica glass preform, which is representative of the surface of the same glass before the drawing.

The 1D power spectral density (PSD) along the drawing direction and the 2D height correlation function extracted from these AFM roughness measurements are shown in Fig. 2. Assuming a sudden freezing of liquid capillary waves at glass transition $[2,37,38]$, the theoretical expression for the PSD for 1D extracted profiles can be derived from Eq. (1) as,

$$
P(f)=\frac{k_{B} T_{G}}{2 \pi \gamma f},
$$

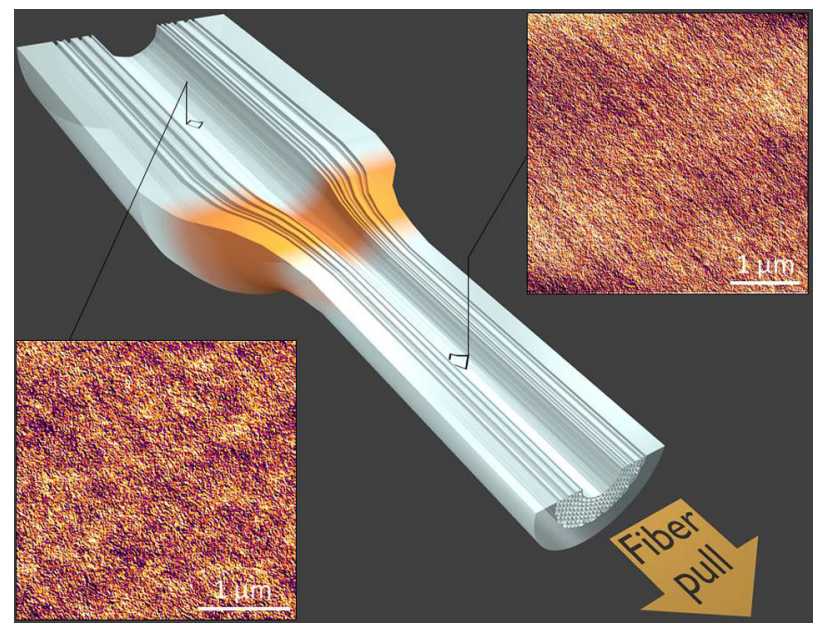

FIG. 1. Sketch of the drawing of hollow core photonic bandgap fibers. The two AFM images represent the surface topography (with the same height range of $1.6 \mathrm{~nm}$ ) of the inner glass surfaces before (preform) and after (fiber) the hot drawing region (in orange). While isotropic before drawing, the subnanometer roughness exhibits clear elongated patterns along the fiber axis after drawing.

where $f$ is the spatial frequency. As illustrated in Fig. 2(a), the predicted $1 / f$ behavior is clearly evidenced in our measurements, and, for the preform, the prefactor is also in excellent agreement with the expected value for silica glass $\left(T_{G}=1500 \mathrm{~K}, \gamma=0.3 \mathrm{~J} \cdot \mathrm{m}^{-2}\right)$ [5]. Surprisingly, while the $1 / f$ behavior also applies to the out-of-equilibrium surfaces of the PBGF fibers, the prefactor is found to be 2 times smaller along the drawing direction. Since the latter controls the final roughness amplitude, this implies that the roughness of the inner interfaces of the fiber is about $\sqrt{2}$ lower than predictions based on thermodynamic noise (at the glass transition temperature $T_{G}$, i.e., in the supercooled state just before freezing), usually expected to represent a lower bound.
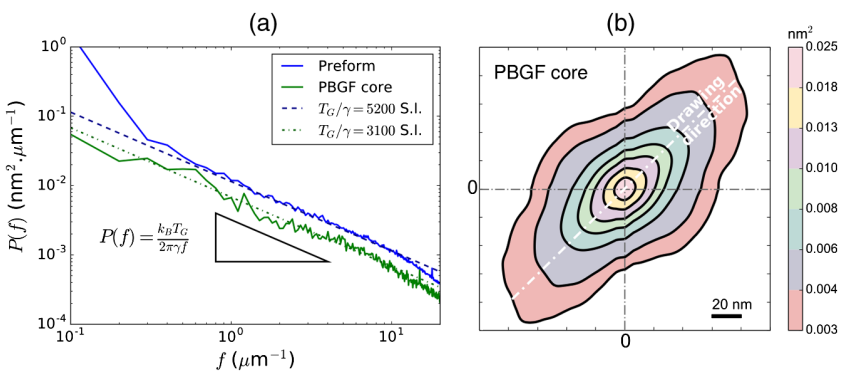

FIG. 2. (a) 1D PSD of the surface roughness along the drawing direction corresponding to the two AFM images of Fig. 1: the inner core surface of the PBGF is much smoother than the reference preform surface. The slope of the triangle illustrates the $1 / f$ evolution from Eq. (2) and the vertical position provides the roughness parameter $T_{G} / \gamma$. (b) The $2 \mathrm{D}$ height autocorrelation of the PGBF surface allows an appreciation of the roughness anisotropy (the AFM scan direction is at $45^{\circ}$ from the fiber axis). 
Another striking feature of the roughness of the PBGF interfaces, illustrated in Fig. 1, is a marked anisotropy. In Fig. 2(b), we give a more quantitative support to this observation: the 2D height autocorrelation functions obtained for the PBGF fiber interfaces are clearly anisotropic with longer range correlations along the drawing direction.

Nonlinear smoothing of driven glass interfaces.-The presence of anisotropic correlations provides strong evidence of an out-of-equilibrium character of the interfaces in the supercooled regime before freezing at the glass transition. Thiébaud and Bickel [8] recently proposed a stochastic hydrodynamic perturbative model of liquid interface under shear flow that predicts attenuation of the capillary modes in the direction of the flow depending on the viscous stress at the interface:

$$
S(\mathbf{q}, \dot{\varepsilon}) \approx \frac{k_{B} T}{\gamma|\mathbf{q}|^{2}}\left[1-A \alpha^{2}\left|\cos \theta_{q}\right|^{2}\right], \quad \alpha=\frac{w_{0} \dot{\varepsilon}}{\gamma / \eta} .
$$

Here, $S(\mathbf{q}, \dot{\varepsilon})$ stands for the surface structure factor in the presence of a shear rate $\dot{\varepsilon}$ ( $\theta_{q}$ gives the angle from the flowing direction), while the prefactor $S_{0}(\mathbf{q})=$ $k_{B} T / \gamma|\mathbf{q}|^{2}$ is the equilibrium expression obtained at a temperature $T$ in the absence of flow. The parameter $\alpha$ is an adimensioned shear rate and $A$ is a numerical constant $[8,9]$. The roughness attenuation depends both on the control parameter $\alpha$ and the direction $\theta_{q}$. In the presence of a flow, the height fluctuations should thus be anisotropic.

The extensional flow of a silica melt under the high thermal gradient that exists during a fiber draw process is obviously far more complex than the shear flow discussed above. Still, numerical models [39] assuming a simple Newtonian behavior of the molten glass reproduce accurately the drawing process of microstructured fibers over a wide range of process parameters (temperature of the furnace, drawing velocity, internal gas pressure) and geometric parameters (air-filling fraction of the fibers). In particular, it appears that the glass membranes defining the microstructure experience high stress during drawing. Vitreous silica in the supercooled regime is indeed characterized by high values of the viscosity $\eta\left(10^{5}-10^{8} \mathrm{~Pa} s\right.$ in the furnace) and strain rates are of the order unity in the hot flowing region so that stress can reach hundreds of MPa.

In the context of an extensional flow, the dimensionless shear rate parameter $\alpha$ can be viewed as a ratio between an attenuation velocity due to stretching and the damping velocity of the capillary waves, i.e., a capillary number [9]. This parameter can also be rewritten in terms of stress or interface tension: $\alpha=w_{0} \sigma_{\text {vis }} / \gamma$, i.e., the ratio of interface tension $\gamma$ with an effective tension $w_{0} \sigma_{\mathrm{vis}}$ induced by the viscous stress $\sigma_{\mathrm{vis}}=\eta \dot{\varepsilon}$ acting across the equilibrium width of the liquid interface $w_{0}$. This definition can be immediately generalized on dimensional grounds to build a control parameter characteristic of the drawing process: $\alpha_{D}=w_{G} \sigma_{M} / \gamma$. Here, $w_{G}=\sqrt{k_{B} T_{G} / \gamma}$ is the glass surface roughness expected in the absence of drawing and $\sigma_{M}$ is the maximum tensile stress experienced by the fiber during the drawing. Numerical modeling of $\sigma_{M}$ according to Ref. [39] shows that the parameter $\alpha_{D}$ can take significant values during the drawing process. This suggests that, against common belief [40], the drawing process may significantly lower the surface roughness within glass microstructured fibers.

Since the viscosity of glasses is strongly dependent on temperature, a change of temperature is expected to induce a large variation of the viscous stress, hence of the parameter $\alpha_{D}$. Therefore, a series of model fibers (hollow capillary tubes) were prepared under different drawing conditions. The furnace temperature was varied in the range $\left[2000^{\circ} \mathrm{C}-2100^{\circ} \mathrm{C}\right]$ while the pulling rate and the geometry were kept constant. A fiber with an outer diameter of $220 \mu \mathrm{m}$ with a thickness of $15 \mu \mathrm{m}$ was obtained by applying a suitable pressure within the capillary during the draw. This allowed us to vary the tensile stress $\sigma_{M}$ in the range [27-114 MPa] and, consequently, the adimensioned control parameter $\alpha_{D}$ in the range [0.024-0.101] (see also Table S1 in the Supplemental Material [24]).

A series of AFM measurements were performed on the inner surfaces of 6 different types of hollow capillary tubes obtained at increasing levels of stress. The results are summarized in Fig. 3 where we plot the rms roughness (height standard deviation) measured over scanned areas of the same size $10 \mu \mathrm{m} \times 10 \mu \mathrm{m}$ [41] vs the drawing stress estimated from the numerical model of drawing [39]. Just as for PBGFs, the surface roughness lies well below that of our reference fire polished preform $\left(\sigma_{M}=0\right)$. Moreover, the roughness appears to decrease as a power law of the drawing stress with an exponent close to $1 / 3$, as emphasized in the inset of Fig. 3.

In addition to the roughness reduction, an evident anisotropic effect can again be identified. As shown on

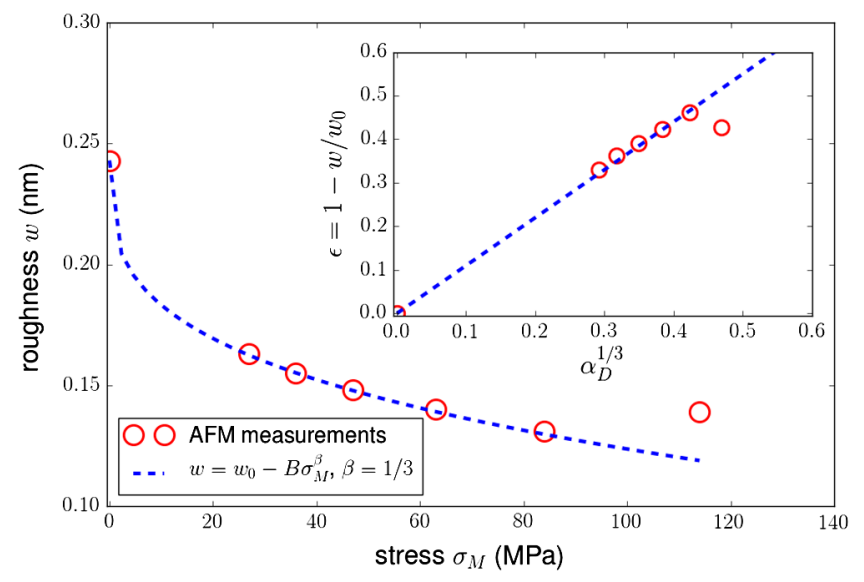

FIG. 3. rms roughness $w$ of inner fiber surfaces obtained at increasing drawing stress $\sigma_{M}$ measured on $10 \times 10 \mu \mathrm{m}^{2} \mathrm{AFM}$ images. Inset: the roughness attenuation $\varepsilon=1-w / w_{0}$ scales as the cubic root of the dimensionless stress $\alpha_{D}$. 
(a)

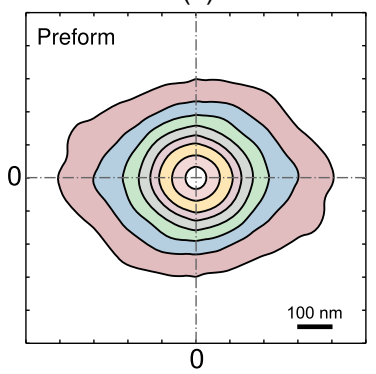

(c)

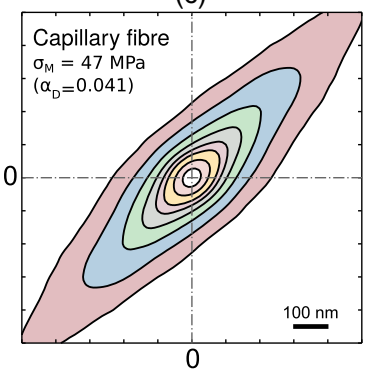

(b)

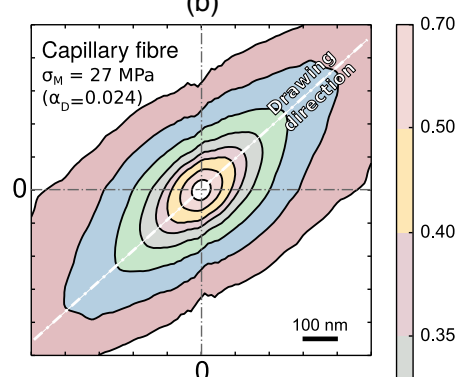

(d)

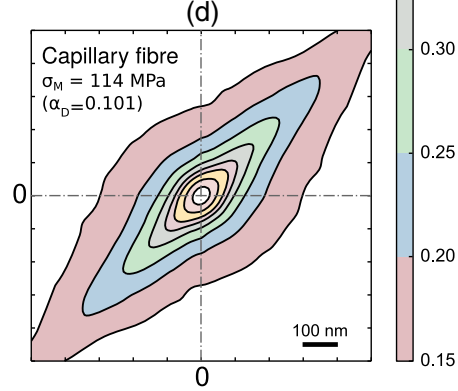

FIG. 4. Normalized 2D height autocorrelation functions of the inner surface roughness of the silica preform (a) and of three hollow capillary fibers obtained by drawing at increasing levels of stress: $\sigma_{M}=27$ (b), $\sigma_{M}=47$ (c), and $\sigma_{M}=114 \mathrm{MPa}(\mathrm{d})$. The degree of anisotropy increases with $\sigma_{M}$ (and the adimensioned control parameter $\alpha_{D}$ ). The AFM scanning direction is at $45^{\circ}$ to the drawing direction to rule out the residual intrinsic anisotropy of the instrument, which is appreciable on the isotropic preform surface shown in (a).

the 2D height correlation maps in Fig. 4, the larger the dimensionless stress $\alpha_{D}$, the more anisotropic the surface roughness. Beyond anisotropy, a stretching effect is also noticed on the 2D correlation functions: the larger $\alpha_{D}$, the more diamondlike the level lines. Note here that in order to eliminate any bias due to the intrinsic anisotropy of the instrument, AFM measurements were performed along directions at $45^{\circ}$ relative to the drawing direction as justified by an extensive metrological investigation reported in the Supplemental Material [24].

Owing to the easier access to the inner surfaces in the capillary fibers with respect to the PBGFs, a fuller range of AFM measurements could be performed and a full quantitative characterization of the two-dimensional surface roughness spectrum was obtained [24]. The scaling behavior expected for frozen capillary waves was again obtained, but with an angular dependent prefactor:

$$
S(\vec{q}) \approx k_{B} A_{F}\left(\theta_{q}\right) /|\vec{q}|^{2} .
$$

As shown in Fig. 5, the prefactor $A_{F}\left(\theta_{q}\right)$, which can be seen as an effective value of the ratio $T_{G} / \gamma$, is systematically lower when measured along the drawing direction than in the azimuthal direction. The observed anisotropy is such that it cannot be described with the simple first order perturbative expansion given by Eq. (3). Nonlinear effects

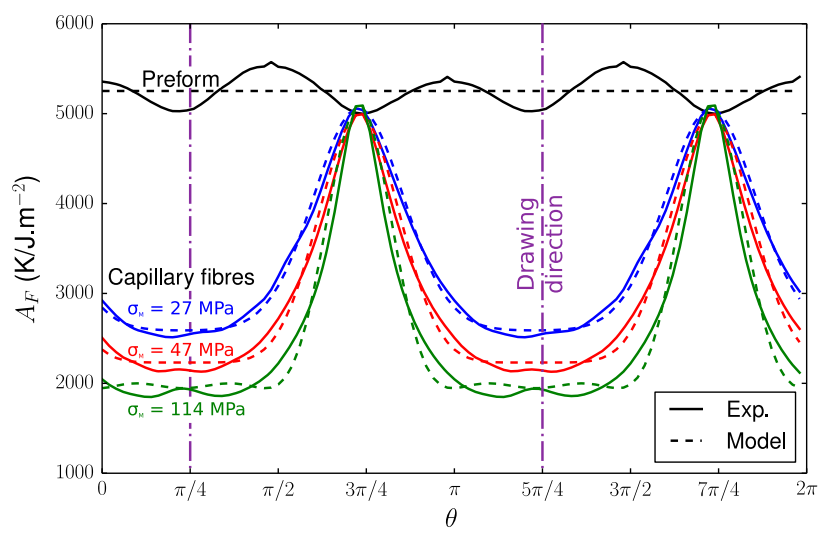

FIG. 5. Angular dependence of the prefactor $A_{F}(\theta)$, i.e., the effective ratio $T_{G} / \gamma$ obtained from the $2 \mathrm{D}$ autocorrelation functions of Fig. 4. While the preform signal presents maxima along the AFM scan axes, the drawn fibers present maxima along the drawing direction (here at $45^{\circ}$ ) and reflect the physical anisotropy of the surface roughness. Dashed lines represent the fits obtained with the analytical expression (5).

are at play and higher mode contributions are significant. Rather than extracting a series of anisotropic contributions [42], we observed here that the nonlinear effects could be captured by accounting for a simple nonlinear transformation of the polar angle: $2 \theta_{q} \rightarrow 2 \theta_{q}+b \sin \left(2 \theta_{q}\right)$. The use of this sine circle map that either contracts or expands the angular metrics depending on the orientation enables us to propose a simple analytic ansatz accounting for the measured surface structure factor of anisotropic frozen capillary waves:

$$
S(\mathbf{q}) \approx \frac{k_{B} T_{G}}{\gamma|\mathbf{q}|^{2}}\left(1-a\left[1+\cos \left(2 \theta_{q}+b \sin 2 \theta_{q}\right)\right]\right) .
$$

Conclusion.-Glass surfaces obtained by drawing thus consist of a superposition of frozen attenuated capillary waves. They result from two successive out-of-equilibrium processes: (i) the nonlinear attenuation of thermal capillary waves in the stretched liquid phase; (ii) the freezing of these attenuated fluctuations when the liquid is quenched through the glass transition. Owing to the combination of these two mechanisms, glass surfaces can retain a memory of the flow direction in the liquid state. The existence of such anisotropic fluctuations has previously been observed and discussed for critical systems [43-45], but never observed to our knowledge in the framework of standard hydrodynamics.

While we report here evidence of a dramatic effect of a flow on the structure of a silica glass surface, early studies were mostly focused on the effect of the stretch rate on the bulk structure of silicate glasses $[46,47]$. Note here that unlike the case of polymers, a flow-induced anisotropy in the structure of amorphous silica cannot result in the long range alignment of (here absent) chainlike structures [48]. Recent experimental and numerical results seem to confirm the emergence of a flow-induced structural anisotropy, but 
limited to the molecular scale [49-51], far smaller than the scale range of frozen capillary waves reported here.

The present experimental results naturally call for further theoretical studies on the effect of an external field on interface fluctuations $[8,44,45]$ and the associated encoding of a memory in the disordered structure [52-54]. In a more applied perspective, the possibility to decrease the surface roughness below the expected lower bound with a fine tuning of the drawing condition is obviously of great interest in the context of the development of photonic band-gap fibers. Moreover, the quantitative characterization of the nonlinear roughness spectrum of the inner interfaces of PBGF should pave the way toward a rigorous computation of scattering losses.

Data underpinning this paper is available from the University of Southampton Institutional repository at [55].

This work was supported by the EU 7th Framework Program under Grant Agreement 228033 (MODE-GAP). This work was funded in part by the UK Engineering and Physical Sciences Research Council through Grants No. EP/P030181/1 (AirGuide Photonics Programme Grant) and No. EP/N00762X/1 (National Hub in High Value Photonic Manufacturing). F. P. and D. J. R. acknowledge the support of the Royal Society. X. B. acknowledges the support of Programme DIM OxyMORE, Ile de France. The authors acknowledge N. K. Baddela and J. Hayes for fabricating the 37 cell HC-PBGF. B. B. and D. V. acknowledge J.-T. Fonné, E. Gouillart, and H. Montigaud at Laboratoire SVI (CNRS/Saint-Gobain) where part of the AFM measurements were performed.

[1] J. Zarzycki, Glasses and the Vitreous State (Cambridge University Press, Cambridge, England, 1991).

[2] J. Jäckle and K. Kawazaki, J. Phys. Condens. Matter 7, 4351 (1995).

[3] T. Seydel, M. Tolan, B. M. Ocko, O. H. Seeck, R. Weber, E. DiMasi, and W. Press, Phys. Rev. B 65, 184207 (2002).

[4] P. J. Roberts, F. Couny, H. Sabert, B. J. Mangan, D. P. Williams, L. Farr, M. W. Mason, A. Tomlinson, T. A. Birks, J. C. Knight, and P. St. J. Russel, Opt. Express 13, 236 (2005).

[5] T. Sarlat, A. Lelarge, E. Søndergård, and D. Vandembroucq, Eur. Phys. J. B 54, 121 (2006).

[6] F. Charru, Hydrodynamic Instabilities (Cambridge University Press, Cambridge, England, 2011).

[7] D. Derks, D. G. A. L. Aarts, D. Bonn, H. N. W. Lekkerkerker, and A. Imhof, Phys. Rev. Lett. 97, 038301 (2006).

[8] M. Thiébaud and T. Bickel, Phys. Rev. E 81, 031602 (2010).

[9] M. Thiébaud, Y. Amarouchene, and T. Bickel, J. Stat. Mech. (2014) P12011.

[10] D. Langevin, Light Scattering by Liquid Surfaces and Complementary Techniques (Marcel Dekker, New York, 1992).

[11] A. Braslau, M. Deutsch, P. S. Pershan, A. H. Weiss, J. Als-Nielsen, and J. Bohr, Phys. Rev. Lett. 54, 114 (1985).
[12] A. Braslau, P. S. Pershan, G. Swislow, B. M. Ocko, and J. Als-Nielsen, Phys. Rev. A 38, 2457 (1988).

[13] M. K. Sanyal, S. K. Sinha, K. G. Huang, and B. M. Ocko, Phys. Rev. Lett. 66, 628 (1991).

[14] A. Madsen, T. Seydel, M. Sprung, C. Gutt, M. Tolan, and G. Grübel, Phys. Rev. Lett. 92, 096104 (2004).

[15] M. Sprung, T. Seydel, C. Gutt, R. Weber, E. DiMasi, A. Madsen, and M. Tolan, Phys. Rev. E 70, 051809 (2004).

[16] D. G. A. L. Aarts, M. Schmidt, and H. N. W. Lekkerkerker, Science 304, 847 (2004).

[17] A. Tay, C. Thibierge, D. Fournier, C. Fretigny, F. Lequeux, C. Monteux, J. P. Roger, and L. Talini, Rev. Sci. Instrum. 79, 103107 (2008).

[18] B. Pottier, G. Ducouret, C. Fretigny, F. Lequeux, and L. Talini, Soft Matter 7, 7843 (2011).

[19] The spectral range of accessible measurements is controlled here by the linear size $L$ of the scanned area, $q_{\min } \approx 2 \pi / L$ and by the radius $a$ of the AFM tip $q_{\max } \approx 2 \pi / a$.

[20] M. C. Phan-Huy, J. M. Moison, J. A. Levenson, S. Richard, G. Mélin, M. Douay, and Y. Quiquempois, J. Lightwave Technol. 27, 1597 (2009).

[21] C. Brun, X. Buet, B. Bresson, M. S. Capelle, M. Ciccotti, A. Ghomari, P. Lecomte, J. P. Roger, F. Poletti, M. Petrovich, D. J. Richardson, D. Vandembroucq, and G. Tessier, Opt. Express 22, 29554 (2014).

[22] X. Buet, C. Brun, B. Bresson, J. Gâteau, S. Sandoghchi, E. N. Fokoua, F. Poletti, D. J. R. M. Petrovich, D. Vandembroucq, and G. Tessier, Opt. Lett. 41, 5086 (2016).

[23] E. N. Fokoua, F. Poletti, and D. J. Richardson, Opt. Express 20, 20980 (2012).

[24] See Supplemental Material at http://link.aps.org/ supplemental/10.1103/PhysRevLett.119.235501 for technical details on the fabrication of fibers and on the AFM measurements, which includes Refs. [25-34].

[25] A. D. Fitt, K. Furusawa, T. M. Monro, C. P. Please, and D. J. Richardson, J. Eng. Math. 43, 201 (2002).

[26] R. H. Doremus, J. Appl. Phys. 92, 7619 (2002).

[27] C. H. Lui, L. Liu, K. F. Mak, G. W. Flynn, and T. F. Heinz, Nature (London) 462, 339 (2009).

[28] A. Labuda, M. Lysy, W. Paul, Y. Miyahara, P. Grütter, R. Bennewitz, and M. Sutton, Phys. Rev. E 86, 031104 (2012).

[29] R. García and R. Pérez, Surf. Sci. Rep. 47, 197 (2002).

[30] L. Nony, R. Boisgard, and J. P. Aimé, J. Chem. Phys. 111, 1615 (1999).

[31] F. Lechenault, G. Pallares, M. George, C. L. Rountree, E. Bouchaud, and M. Ciccotti, Phys. Rev. Lett. 104, 025502 (2010).

[32] P. R. Nayak, Wear 26, 165 (1973).

[33] A. Duparré, J. Ferre-Borrull, S. Gliech, G. Notni, J. Steinert, and J. M. Bennett, Appl. Opt. 41, 154 (2002).

[34] E. Dura, J. Bell, and D. Lane, IEEE Journal of Oceanic Engineering 33, 434 (2008).

[35] Inner surfaces were chosen because they are protected from contaminants and directly interact with light propagating within such fibers, which make them particularly important in the context of HC-PBGFs.

[36] F. Poletti, M. N. Petrovich, and D. J. Richardson, Nanophotonics 2, 315 (2013).

[37] Y. Chushkin, C. Caronna, and A. Madsen, Europhys. Lett. 83, 36001 (2008). 
[38] In absence of flow, the roughness spectrum extracted from our AFM measurements is consistent with the Maxwell Debye model proposed in Ref. [2] to describe the freezing of capillary waves. In contrast with Ref. [37] no dependence on low frequency elasticity was observed.

[39] G. T. Jasion, J. S. Shrimpton, Y. Chen, Bradley, D. J. Richardson, and F. Poletti, Opt. Express 23, 312 (2015).

[40] J. Roberts, H. Sabert, B. J. Mangane, and L. Farr, Hollow core optical fiber and making same, U.S. Patent No. US 7, 366,388 B2 (2008).

[41] The scaling of the spectrum of capillary waves $S(q) \propto 1 / q^{2}$ is such that the variance of the height depends on the bounds of the spectral range and scales roughly as $\log \left(q_{\min } / q_{\max }\right)$. In order to make comparisons between different protocols of preparation, it is thus crucial to estimate the roughness of regions of the same size.

[42] I. Arad, B. Dhruva, S. Kurien, V. S. L'vov, I. Procaccia, and K. R. Sreenivasan, Phys. Rev. Lett. 81, 5330 (1998).

[43] D. Beysens, M. Gbadamassi, and L. Boyer, Phys. Rev. Lett. 43, 1253 (1979).

[44] A. Onuki and K. Kawasaki, Ann. Phys. (N.Y.) 121, 456 (1979).
[45] T. H. R. Smith, O. Vasilyev, D. B. Abraham, A. Maciolek, and M. Schmidt, Phys. Rev. Lett. 101, 067203 (2008).

[46] T. Takamori and M. Tomozawa, J. Am. Ceram. Soc. 59, 377 (1976).

[47] J. F. Stebbins, D. R. Spearing, and I. Farnan, J. Non-Cryst. Solids 110, 1 (1989).

[48] P. J. Miller, G. J. Exarhos, and W. M. R. Jr., J. Chem. Phys. 59, 2796 (1973).

[49] C. L. Rountree, D. Vandembroucq, M. Talamali, E. Bouchaud, and S. Roux, Phys. Rev. Lett. 102, 195501 (2009).

[50] J. Wu, J. Deubener, J. F. Stebbins, L. Grygarova, H. Behrens, L. Wondraczek, and Y. Yue, J. Chem. Phys. 131, 104504 (2009).

[51] T. Sato, N. Funamori, and T. Yagi, J. Appl. Phys. 114, 103509 (2013).

[52] K. Sekimoto, Physica (Amsterdam) 170A, 150 (1990).

[53] N. C. Keim and S. R. Nagel, Phys. Rev. Lett. 107, 010603 (2011).

[54] D. Fiocco, G. Foffi, and S. Sastry, Phys. Rev. Lett. 112, 025702 (2014).

[55] https://doi.org/10.5258/SOTON/D0290. 